\title{
Estimación de la Carga de Transformadores de Potencia Utilizando una Red Neuronal Artificial
}

\author{
Laura Agudelo $^{(1)}$, Esteban Velilla ${ }^{(2)}$ y Jesús M. López ${ }^{(2)}$ \\ (1) Interconexión Eléctrica SA (ISA), Calle 12 Sur No. 18 - 168 Bloque 2,Medellín, Colombia (e- \\ mail:laura.agudelo@gmail.com) \\ (2)Grupo de Manejo Eficiente de la Energía - GIMEL, Departamento de Ingeniería Eléctrica, Facultad de \\ Ingeniería, Universidad de Antioquia UdeA, Calle 70No. 52-21,Medellín, Colombia (e-mail: \\ esteban.velilla@gmail.com; lezama@udea.edu.co)
}

Recibido Oct. 7, 2013; Aceptado Dic. 10, 2013; Versión final recibida Ene. 12, 2014

\begin{abstract}
Resumen
En este artículo se presenta una metodología para estimar la curva de carga de transformadores de potencia utilizando redes neuronales artificiales. Para implementar la metodología propuesta se utilizaron datos reales de dos transformadores ubicados en diferentes zonas geográficas y con diferentes condiciones de operación. La técnica de estimación de carga fue implementada con datos históricos de la empresa Interconexión Eléctrica S.A (ISA).Para realizar la predicción de las curvas de carga se entrenó una red neuronal usando MATLAB, la cual se adapta a la curva de carga con tiempos de predicción diaria y semanal. La metodología propuesta permite la estimación de la curva de carga de transformadores de potencia con un error relativo de alrededor del 10\%. Este método puede ser utilizado en otros equipos con características de operación similares.
\end{abstract}

Palabras clave: curva de carga, transformadores de potencia, redes neuronales artificiales

\section{Load Estimation of Power Transformers using an Artificial Neural Network}

\begin{abstract}
This paper presents a methodology for load estimation of power transformers by means of artificial neural networks. To implement the proposed methodology the data of two power transformers, located in different geographical areas and with different operational conditions, were considered. The load estimation methodology was implemented using historical data provided by the company Interconexión Eléctrica S.A. (ISA). To predict the load curves a neural network was trained using MATLAB, being able to fit a load curve with daily and weekly prediction times. The proposed method allows the estimation of load curve values in power transformers with an average percent relative error of around $10 \%$. The method described in this paper can be applied to other equipment with similar operating characteristics.
\end{abstract}

Keywords: electric load curve, power transformers, artificial neural networks 


\section{INTRODUCCIÓN}

Los sistemas eléctricos de potencia son cada vez más importantes en las regiones, tanto en el ámbito social como en el económico, esto ha convertido a la energía eléctrica en un eje estratégico de desarrollo mundial, de ahí la importancia en la implementación y actualización de herramientas tecnológicas que permitan garantizar el suministro de la energía eléctrica en todo momento, para evitar demandas no atendidas.

Para las empresas de transmisión es importante conocer la curva de carga de sus transformadores de potencia, en especial de aquellos que se encuentran operando cerca a sus límites máximos operativos, los que atienden clientes específicos o directamente una carga residencial. La predicción de carga de los transformadores de potencia permite conocer la demanda y garantizar el suministro de energía eléctrica en el corto, mediano y largo plazo. Además, permite realizar estudios de cuantificación de pérdidas mediante acciones operativas complementarias, ayudando a reducir el pico de la demanda. De esta forma, se puede mejorar el uso de los transformadores en tiempo real al optimizar los recursos disponibles, realizando una correcta gestión de los picos de carga.

La intención de estimar o predecir la curva de carga de los transformadores de potencia es prever y adaptarse a la demanda en los distintos plazos. A largo plazo, para dar señales tempranas a los entes reguladores para la adquisición y puesta en servicio de nuevos equipos; a corto plazo, para la operación del sistema en tiempo real. Lo anterior contribuye a garantizar el continuo suministro de energía eléctrica al usuario final (Schau y Novitskiy, 2008). Otra finalidad es la de realizar una correcta gestión de la demanda, es decir, inducir a los consumidores a modificar sus pautas de consumo para lograr una mayor eficiencia conjunta en el proceso de suministro y uso de la energía eléctrica (Wang et al., 2011).

La predicción de curvas de carga en los transformadores de potencia, implicaría altos costos si se realizaran mediciones directas y procesamiento de información por separado a cada equipo, a fin de realizar un análisis riguroso que brinde resultados de alta precisión. Sin embargo, la predicción de curvas de carga, usando técnicas computacionales de inteligencia artificial, permite generalizar y caracterizar los parámetros más significativos que influyen en la curva, para luego aplicarlos a familias de equipos.

Entre los métodos disponibles para la estimación de carga están los de medición directa y los basados en inteligencia artificial. Los métodos de medición directa registran los valores en cada equipo y mediante un tratamiento estadístico de los datos, usan la media y desviación estándar para obtener una curva de carga típica para cada equipo. Sin embargo, esta técnica no permite ser aplicada a una familia de equipos, sino a un equipo específico. Como una variación a este método se tiene la agregación de curvas, en el cual se toman las curvas de carga de los usuarios conectados a un transformador y se clasifican los datos por tipo de cliente (se dividen en sub-categorías), finalmente se caracterizan los usuarios y se aplica el método a otras familias de equipos, según el tipo de cliente. Este método es recomendado para empresas de distribución que no posean equipos ni software robusto de computación (Gallego et al., 2007). Entre los métodos de inteligencia artificial se encuentran la Lógica Difusa y las Redes Neuronales Artificiales (RNA). Para el primero, el diseño de reglas necesarias para una buena inferencia en la predicción, no suele ser obtenido fácilmente (Pérez y Vernon, 2005). En cuanto a las RNA, al ser un modelo no lineal de series temporales, ha aumentado considerablemente su uso en los últimos años, cobrado gran importancia en este campo de investigación y mostrando gran capacidad en la predicción de múltiples aplicaciones (Gonzales et al., 2008).

Las RNA son modelos matemáticos que tratan de imitar las habilidades del cerebro humano, ofreciendo ventajas en cuanto a su capacidad de aprendizaje, generalizando situaciones a partir de casos de estudio, funcionamiento en paralelo y tolerancia a fallos por información imprecisa (Haykin, 1994). Los modelos con RNA son preferiblemente utilizados cuando no se tiene un conocimiento previo del comportamiento de las variables analizadas, pero existen mediciones, observaciones, registros, datos, etc. Algunas de las aplicaciones con RNA están relacionadas con el pronóstico delos precios de la energía (Villada et al., 2011), pronóstico de los precios en mercados de valores (Molina et al., 2012), protección de generadores (Villada et al., 2006),clasificación (Velilla et al., 2009), predicción de series temporales (Barrientos et al., 2012), diseño de puestas a tierra (Salam et al., 2006) y reconocimiento de patrones (Meireles et al., 2003), entre otras.

Debido a que la empresa Interconexión eléctrica S.A (ISA), posee gran cantidad de registros de información y realiza un constante monitoreo a sus transformadores, en este trabajo se optó por la utilización de los modelos de RNA para la estimación de las curvas de carga de 2 transformadores de potencia. Lo anterior se justifica en el hecho de que las RNA son modelos no lineales que se adaptan fácilmente a la representación de diversos fenómenos físicos y adicionalmente, funcionan muy bien cuando se tienen registros a pesar de que existan imperfecciones en la información (Meireles et al., 2003). 


\section{CARACTERIZACION DE LA CURVA DE CARGA}

La caracterización de curvas de carga permite evaluar la tendencia de la demanda durante las 24 horas del día en una semana. Esto con el fin de identificar y caracterizar el patrón de comportamiento según el día de la semana y el tipo de cliente conectado; ya sea residencial, comercial o industrial. El tipo de cliente residencial se caracteriza por mantener una carga variable durante el día, iniciando su consumo en las horas de la mañana y con un pico de demanda pronunciado en las horas de la noche, entre las 18 y 21 horas. El cliente comercial posee un consumo constante empezando en las horas de la mañana, alrededor de las 9 horas, y finalizando a las 22 horas, y se clasifica de acuerdo al tipo de actividad comercial. El cliente industrial tiene un tipo de curva muy variada que depende del tipo de actividad y de la gestión interna del manejo de energía; es decir, si posee o no generación propia para regular el consumo de energía de la red de acuerdo a la hora del día.

Una curva típica de carga de los transformadores (A y B), cuyas especificaciones técnicas se encuentran en la Tabla 1, son presentadas en la Fig. 1 y Fig. 2. La Fig. 1 ilustra la tendencia de la carga del transformador A durante las 24 horas del día, observándose que los días ordinarios conservan una tendencia muy similar, mientras que en la curva para los días sábados y domingos se presenta una disminución de la carga. Esta tendencia se caracteriza por dos patrones de comportamiento: en la mañana aumenta la carga dado su carga comercial e industrial y en la noche se presenta un pico de demanda debido a la carga residencial. En la Fig. 2 se ilustra la carga para el transformador B, en la cual se observa una alta incidencia de carga tipo residencial, ya que se eleva el consumo de demanda entre las 18 y 21 horas; y se obtiene un comportamiento similar para los días sábados y domingos al presentado en el transformador $\mathrm{A}$.

Tabla 1: Características técnicas de los transformadores

\begin{tabular}{|l|c|c|}
\hline Transformador & A & B \\
\hline Potencia (MVA) & 168 & 150 \\
\hline Tensión nominal (kV) & $230 / 115$ & $500 / 110$ \\
\hline Corriente nominal (A) & $422 / 844$ & $173 / 786$ \\
\hline Ubicación & Costa & Oriente \\
\hline Tipo de carga & Residencial, comercial e industrial & Residencial y comercial \\
\hline
\end{tabular}

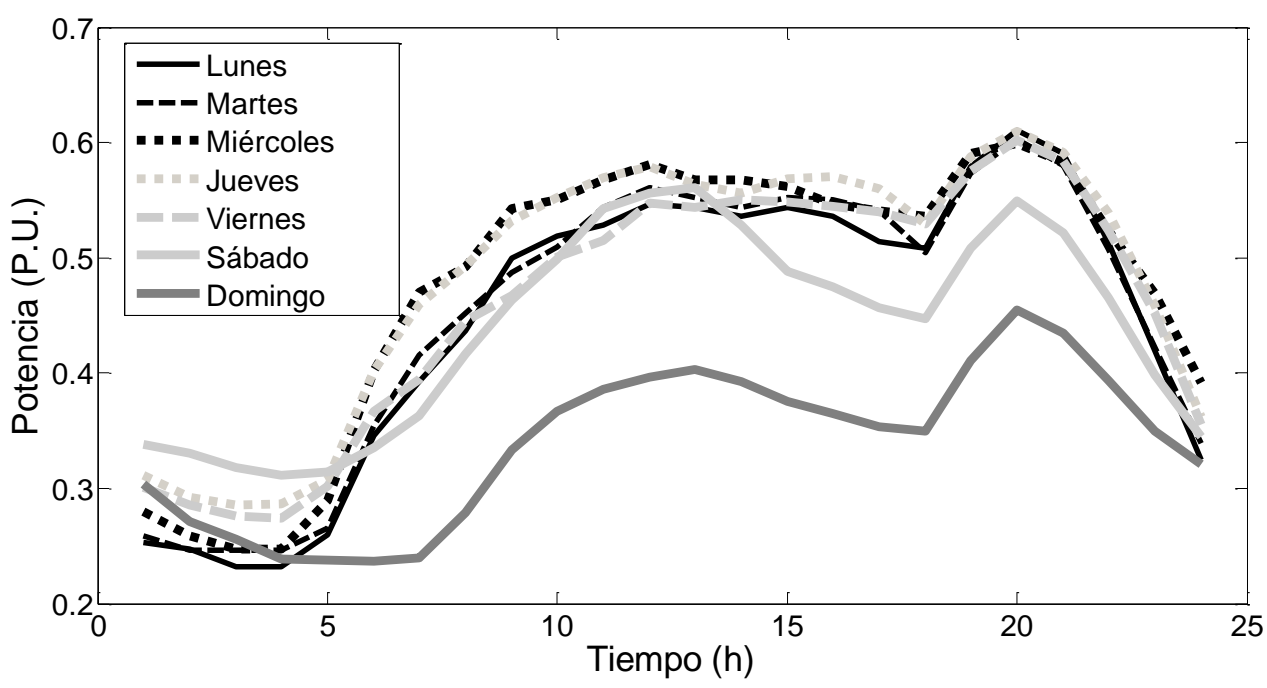

Fig. 1: Curva de carga para el transformador $\mathrm{A}$ 


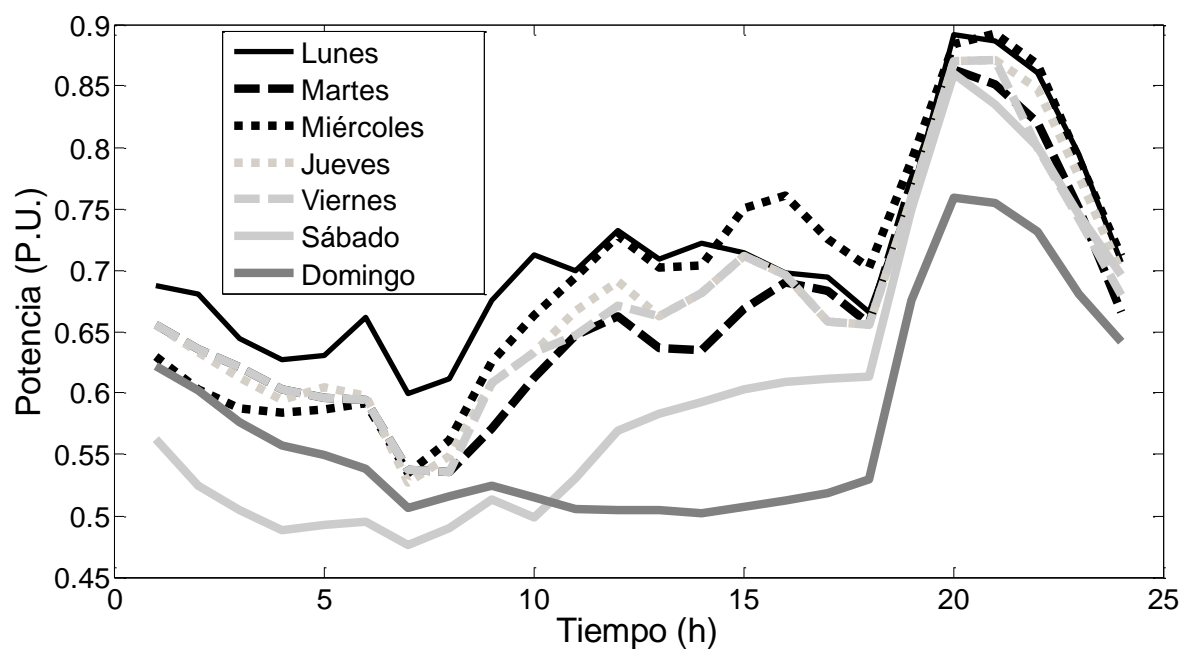

Fig. 2: Curva de carga para el transformador B

Otra forma de observar los datos de Fig. 1 y Fig. 2 es a través de la distribución de datos mediante cuartiles (Fig. 3 y Fig. 4), en estos gráficos el ancho de las barras muestra la variabilidad de los datos con respecto a la mediana, observándose que el transformador B a pesar de tener algunos datos por fuera del tercer cuartil en los días lunes, martes, jueves y viernes (datos atípicos), presenta una simetría general en las barras a lo largo de la semana, excepto el domingo; lo cual indica una distribución de datos más uniforme, o una curva más plana como se observa en la Fig. 2.

En el caso de las curvas de transformadores, algunas variables que afectan la tendencia de la curva de carga son: las condiciones meteorológicas, las cuales perturban el comportamiento y consumo de la energía, especialmente en países con estaciones donde se presentan cambios continuos de temperatura dependiendo la época del año; el calendario, se refiere básicamente al mes y día del año, este factor es importante teniendo en cuenta que hay meses que por su naturaleza afectan el consumo, tales como diciembre y enero (es importante mencionar que las festividades o celebraciones especiales, también cambian la tendencia de la carga); indicadores económicos, estos factores afectan la carga a largo plazo y tienen que ver con el crecimiento de la población y comportamiento en general de la economía en la región; y finalmente factores no predecibles o atípicos en la operación como ocurrencias forzadas, fallas en los equipos de medida o mantenimientos.

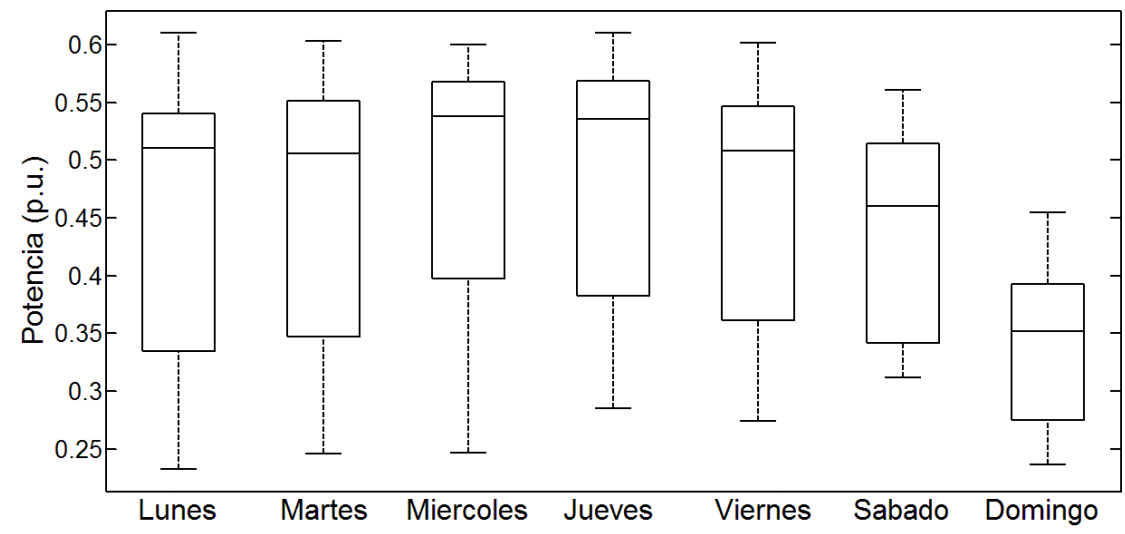

Fig. 3: Distribución de los cuartiles de los datos para el transformador A 


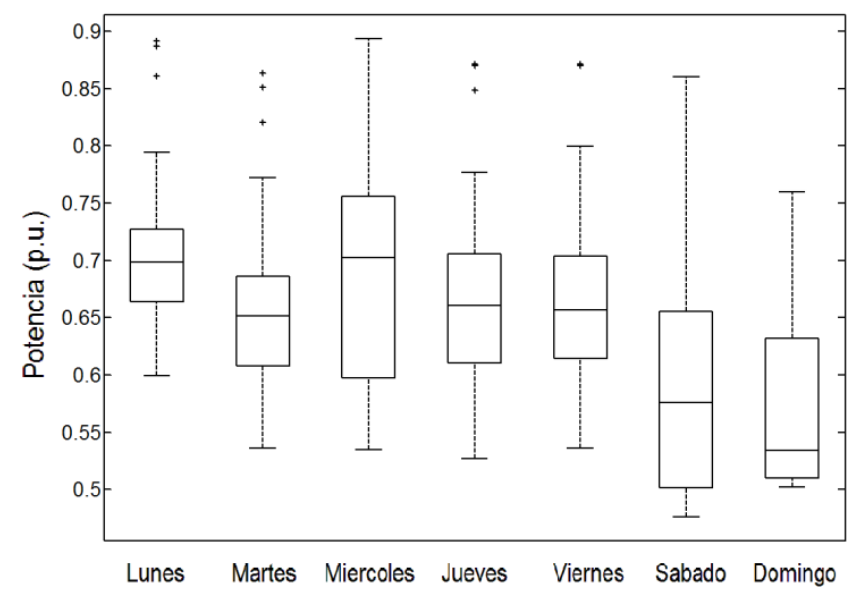

Fig. 4: Distribución de los cuartiles de los datos para el transformador $B$

\section{REDES NEURONALES ARTIFICIALES (RNA)}

En este trabajo para la predicción de curvas de los transformadores de potencia, se usaron RNA debido a que estas presentan una buena capacidad de adaptación a problemas no lineales y tienen eficiente desempeño ante gran volumen de información, lo anterior considerando que las variables que pueden afectan el comportamiento de la curva de los transformadores, suministran información imprecisa o datos no lineales para el análisis.

\section{Modelo de RNA}

Una arquitectura de RNA generalmente utilizada consta de 2 capas: la capa oculta, la cual recibe la información provenientes de las entradas y la capa de salida encargada de entregar la información procesada (Haykin, 1994). Estas 2 capas están conformadas por neuronas, las cuales procesan la información a través de las funciones de activación, para producir la salida de las neuronas y por ende de las diferentes capas existentes en la estructura. Las neuronas de las diversas capas se conectan entre sí, y dependiendo de estas conexiones es que se establece el aprendizaje o memorización de la información (Stanley, 1990).

La estructura de la red neuronal seleccionada fue una feedforward supervisada de 2 capas (una capa oculta y otra de salida).Tomándose 26 variables de entrada: la primera variable es el tipo de día, ordinario, sábado o domingo, los cuales se codificaron como 1, 2 ó 3 respectivamente; la segunda variable es la clase de día, que puede ser laboral o no laboral, o sea, 0 ó 1 respectivamente. Como día no laboral se clasificaron los domingos y días feriados diferentes a este. Las restantes 24 variables corresponden a las corrientes promedio registradas cada hora durante un día. La variable de salida es la potencia de cada transformador.

La base de datos con las variables mencionadas fue tomada de los registros medidos y almacenados por la empresa Interconexión Eléctrica SA (ISA).Los datos se tomaron desde el 01 de enero de 2010 hasta el 30 de mayo de 2012, para una total de 877 muestras. Estos datos se dividieron aleatoriamente en 3 conjuntos, uno para entrenamiento, y otros 2 para validación (observación del aprendizaje) y prueba del modelo. Para el entrenamiento se utilizó el algoritmo Levenberg-Marquardtcon una tasa de aprendizaje de 0.01 (Hankyn, 1994), y como parámetro de parada se tomó de referencia el error medio cuadrático dado por la expresión (1).

$E_{m s e}=\frac{1}{N} \sum_{i=1}^{N}\left(t_{i}-y_{i}\right)^{2}$

Donde $t_{i}$ es el resultado deseado (target), $y_{i}$ es el valor calculado por la RNA y $N$ es el número de datos utilizados. Para determinar el número de capas ocultas se realizaron varias simulaciones para determinar la cantidad óptima de estas, obteniendo con 10 neuronas en la capa oculta, con la cual se obtuvo un error medio cuadrático de 0.0038 . 
Para la predicción de curvas de carga fueron implementados dos modelos: diario y semanal. El modelo diario o corto plazo usa como datos de entrada las corrientes del día anterior; es decir, para predecir la curva de carga del día martes, utiliza los datos del día lunes para el entrenamiento de la red, y así para los demás días. Para el modelo semanal, los datos de entrada para predecir la curva de la semana $\mathrm{n}$, son las corrientes de la semana $n-1$.

\section{PRUEBAS Y RESULTADOS}

Para el transformador A los resultados obtenidos en las simulaciones del modelo diario, se muestran en la Fig.5. Se comparan los datos reales de carga y los datos obtenidos en la simulación de estimación de carga diaria. Para este análisis se evalúan los resultados de cuatro días diferentes: sábado 14 de Julio (a), domingo 15 de Julio (b), lunes 16 de Julio (c) y martes 17 de Julio (d). En todos los casos se utilizaron los datos del día anterior, esto con el fin de observar el comportamiento de la predicción teniendo en cuenta que cada día tiene una caracterización diferente, excepto la estimación que se realizó para el martes con los datos del lunes, días con igual caracterización; es decir, ambos días son ordinarios y laborables.

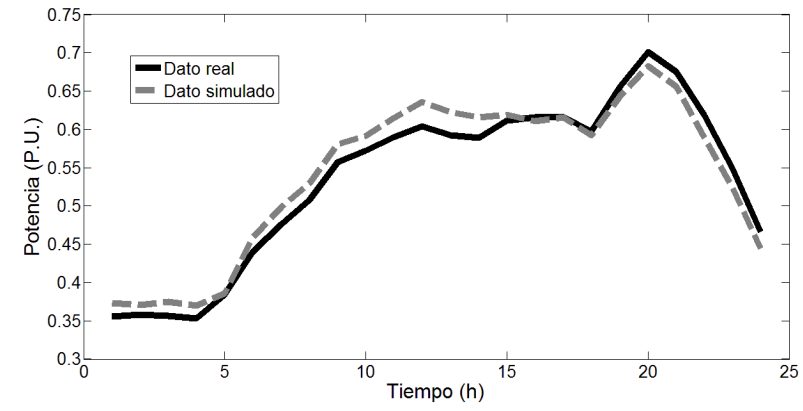

a) Sábado (Julio 14)

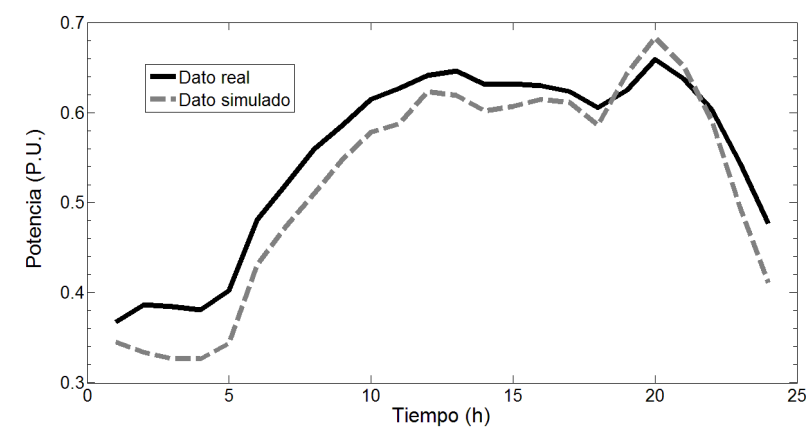

c) Lunes (Julio 16)

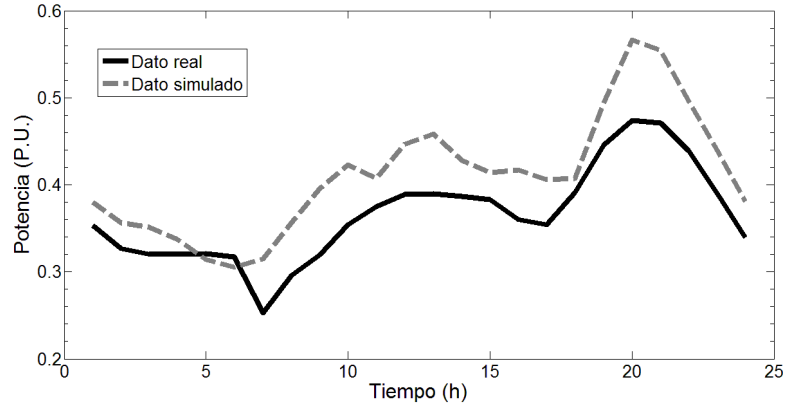

b) Domingo (Julio 15)

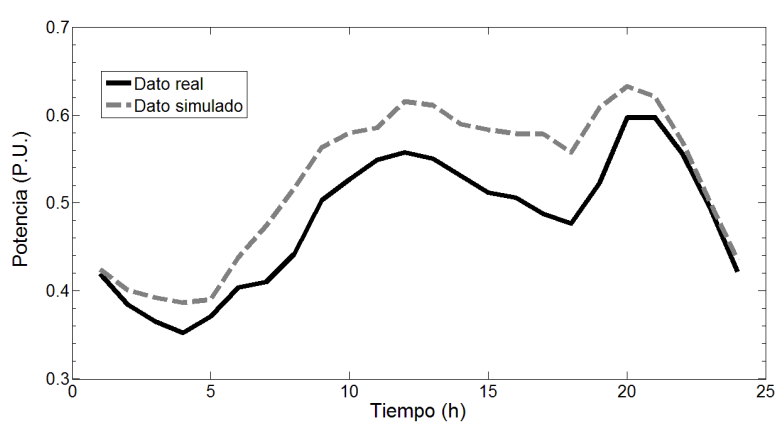

d) Martes (Julio 17)

Fig. 5: Carga Real vs Estimada diaria del Transformador A

En general, se obtuvo una buena estimación para los días simulados, en la predicción del día martes (ordinario) se consiguió la mejor estimación de carga con errores relativos porcentuales (ERP) entre los datos menores al $5 \%$ y en la predicción del día domingo se obtuvo el mayor ERP del $15 \%$, debido a que durante el entrenamiento se tenían más datos de días ordinarios que festivos.

En la Fig. 6 se ilustra la comparación entre la estimación de carga del modelo semanal, incluyendo los días ordinarios y fines de semana del 9 al 15 de Julio de 2012. Al igual que en los resultados anteriores se puede apreciar una buena estimación de carga en los días ordinarios y sábado con ERP menores al $10 \%$, mientras que el día domingo, aunque se conserva la tendencia en la curva, existe diferencia del ERP cercanos al $15 \%$. 


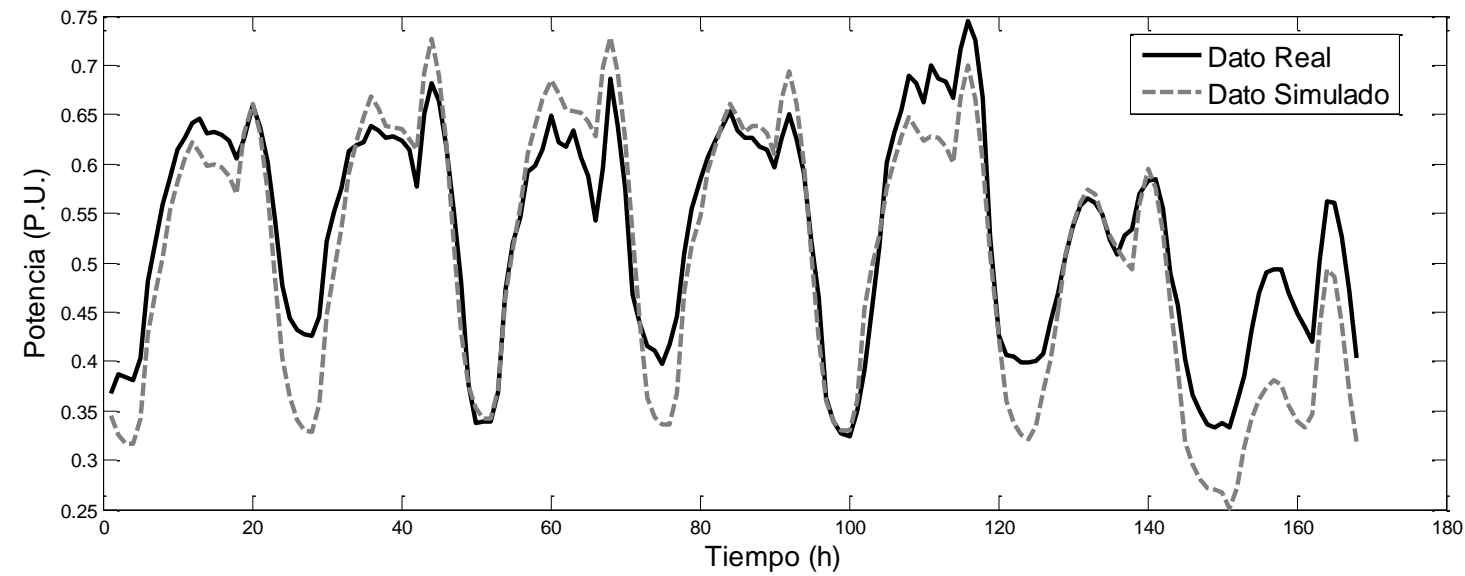

Fig. 6: Carga Real vs Estimada para la semana (Julio 9 a 15) del Transformador B

En la Fig. 7 se ilustran los resultados de simulación de estimación de carga del transformador B, usando el modelo diario. Para analizar los resultados y los errores relativos porcentuales entre los datos reales y simulados se realizó una comparación entre estos. Al igual que para el caso anterior, se tomaron cuatro días como referencia para el estudio: sábado 02 de Junio (a), domingo 03 de Junio (b), lunes 04 de Junio (c) y martes 05 de Junio (d), para todos los casos fueron usados los datos del día anterior para realizar el entrenamiento. De los datos estudiados se obtuvo una buena estimación para los días simulados con una precisión en los resultados mayor al $88 \%$, en la predicción del día sábado se obtuvo la mejor estimación de curva de carga con ERP entre los datos menores al 6\%, en la predicción del día domingo se obtuvo el mayor ERP del $12 \%$ entre algunos datos.

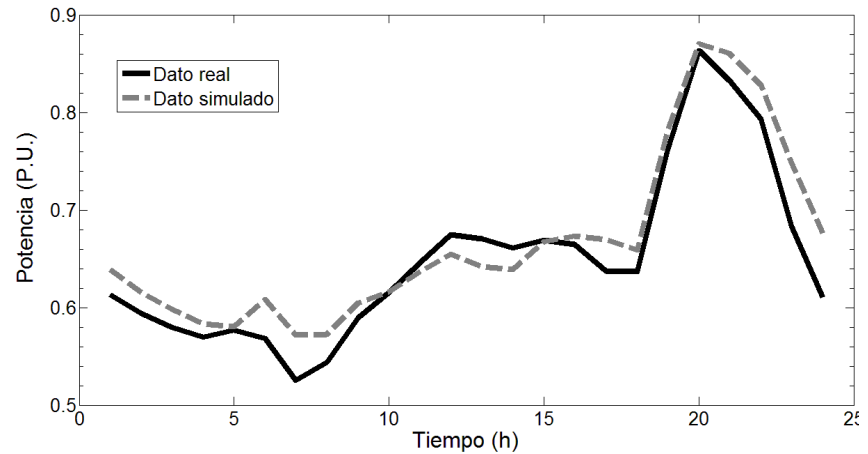

a) Sábado (Junio 02)

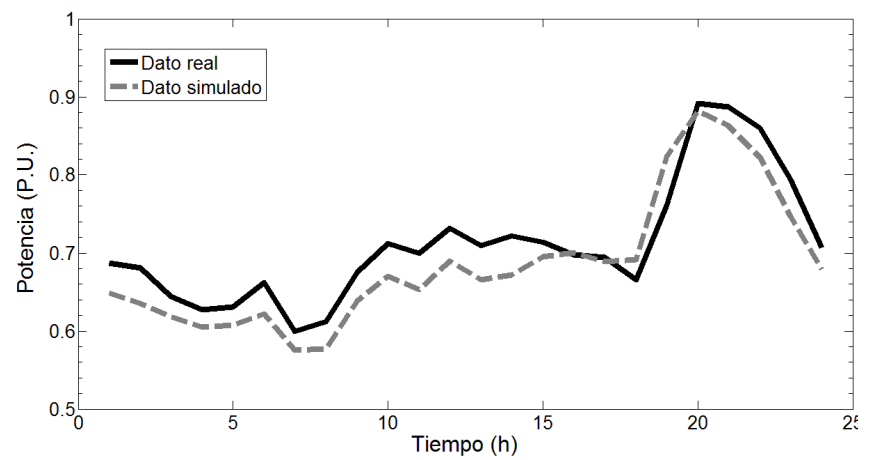

c) Lunes (Junio 04)

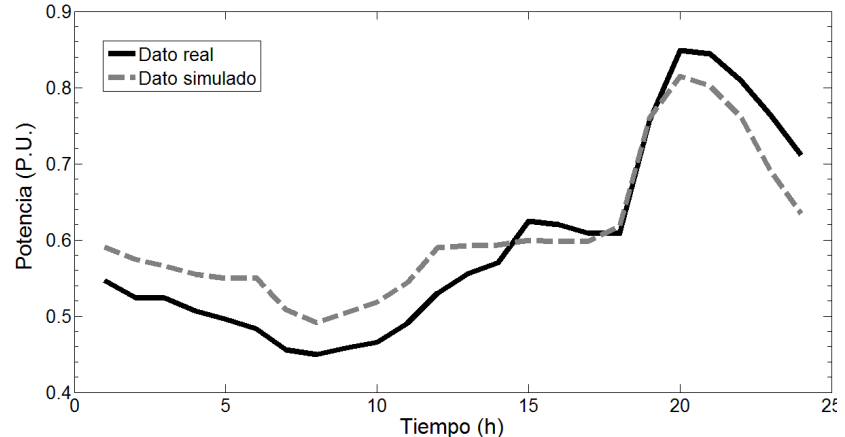

b) Domingo (Junio 03)

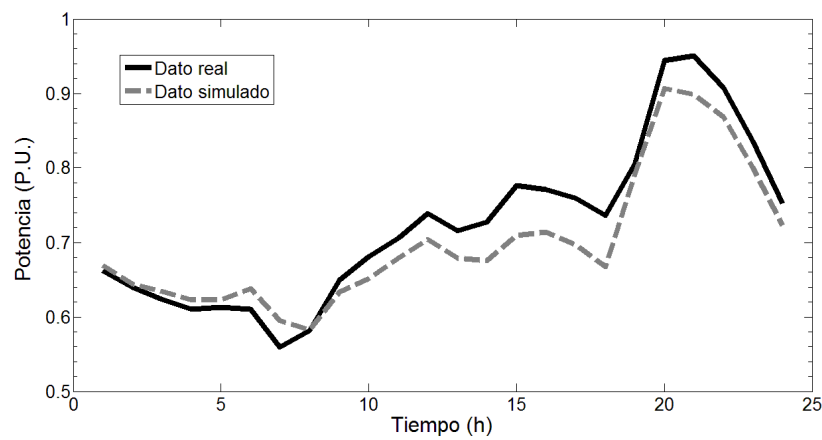

d) Martes (Junio 05)

Fig. 7: Carga Real vs Estimada diaria del Transformador B

Finalmente en la Fig. 8, calculamos la estimación de la curva de la carga del transformador B para el modelo semanal, del 18 al 24 de junio de 2012, vemos que podemos tener una tendencia aproximada a partir de datos de la semana anterior, incluso para sábados y domingos; en la madrugada del día jueves se observa 
una variación en la tendencia de los datos reales, lo anterior se debe a una condición atípica en la operación del transformador, es posible que se haya presentado una pérdida o baja en la demanda de un cliente especifico, por tanto en ese punto se obtuvo un ERP cercano al $20 \%$, en los demás datos el ERP estuvo alrededor del $5 \%$.

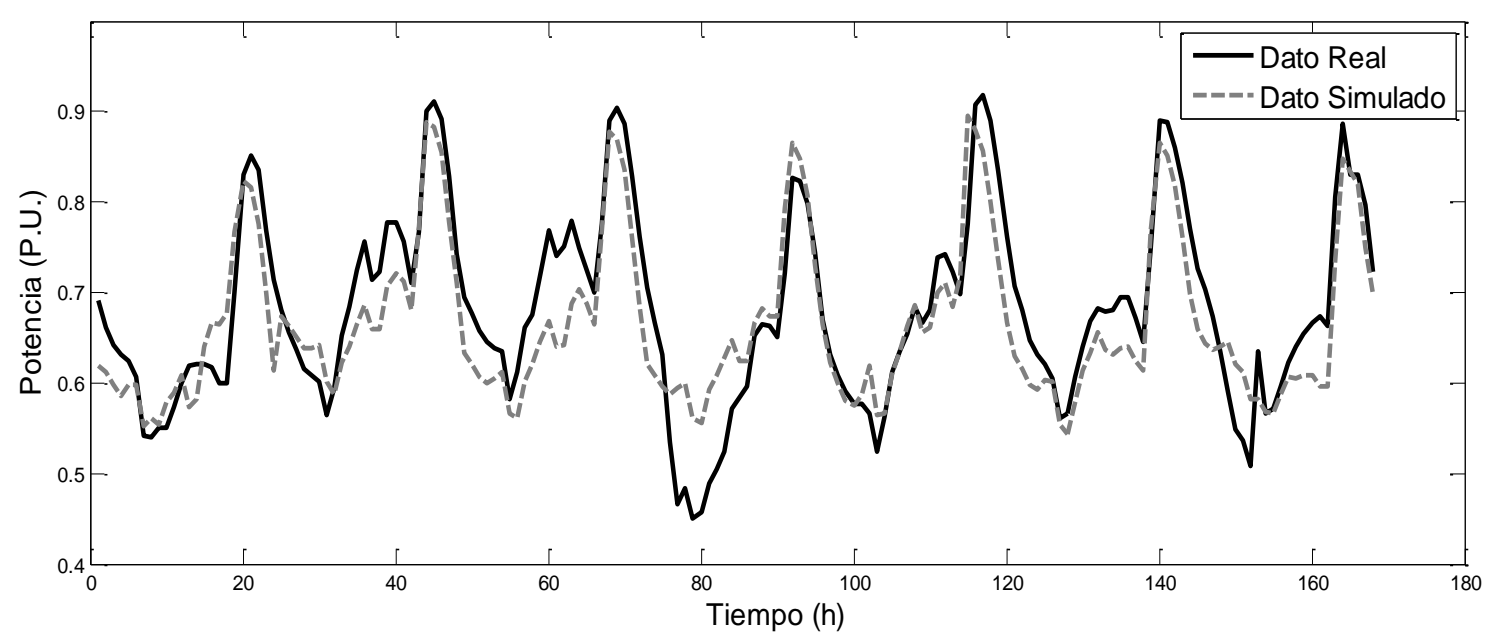

Fig. 8: Carga Real vs Estimada para la semana (Junio 18 a 24) del Transformador B.

\section{CONCLUSIONES}

De los resultados obtenidos al entrenar la RNA, se observa que ambos modelos, diario y semanal, presentan un buen comportamiento en la predicción de la curva de carga los transformadores de potencia para los días ordinarios, mientras que los ERP para los días festivos fueron los más altos, esto debido a que la base de datos con la que se realizó el entrenamiento tenía más datos de días ordinarios que festivos.

Es importante caracterizar las curvas de carga ya sea según su ubicación, tipo de carga conectada o nivel de tensión, esto permite identificar condiciones operativas atípicas y dar alertas tempranas en caso de que se requieran.

En aquellos equipos que operan cerca a sus valores nominales como es el caso del transformador B, es importante realizar una eficiente gestión de la carga en periodos de máxima demanda entre las 18 y 21 horas a fin de garantizar la continuidad y confiabilidad en el servicio al usuario final. Se deben analizar e implementar estrategias en conjunto con el usuario final que busquen optimizar los recursos, por ejemplo desplazando el aumento de la carga desde las 16 horas y así disminuyendo el pico de carga entre las 18 y 21 horas, para evitar el riesgo de operar en sobrecarga.

Se propone seguir mejorando la clasificación de los datos de entrada a la red neuronal, a fin de obtener una mejor predicción en la curva de carga, especialmente para los días domingo y feriados, evaluando la pertinencia de asignar dentro de las entradas datos como: mes, día, temperatura, tipo de carga, entre otros.

La iniciativa de la investigación fue académica; sin embargo, se pretende para desarrollos futuros aplicar la metodología para trabajar en la predicción de curvas de carga de transformadores de potencia en escenarios mensuales (mediano plazo) y anuales (largo plazo) e integrarlas a las herramientas de planeamiento energético existentes.

\section{AGRADECIMIENTOS}

Los autores expresan sus agradecimientos a la empresa Interconexión Eléctrica S.A. y al Proyecto de Sostenibilidad 2013-2014 de la Universidad de Antioquia por el apoyo técnico y económico recibido para el desarrollo de este proyecto.

\section{REFERENCIAS}

Barrientos, J., E. Rodas, E. Velilla, M. Lopera y F. Villada. Modelo para el pronóstico del precio de la energía eléctrica en Colombia. Revista Lecturas de Economía: 77(1), 91-127 (2012) 
Gallego L. A., A. Francisquini, O. Gómez, O y A. Padilha A., Estimación de curvas de demanda de consumidores, transformadores de distribución y alimentadores primarios en sistemas de distribución. Revista Scientia e Technica. 13 (35), 107-112. (2007).

Gonzalez X., A. Perea., G. Caicedo y F. Castro., Estimación de curvas de carga en transformadores eléctricos mediante redes neuronales. Revista Ingeniería y Competitividad: 10 (2), 75-86 (2008).

Haykin, S., Neural Networks: A comprehensive Foundation, $1^{\text {a }}$ edición, Macmillan College Publishing Company. (1994).

Meireles. M.R.G., P.E.M Almeida y M.G Simoes., A comprehensive Review for Industrial Applicability of Artificial Neural Networks: IEEE Transactions on Industrial Electronics: 50 (3), 585-601 (2003).

Molina, J.D., F. Villada y García E.,Pronóstico del precio de la energía eléctrica usando redes neuro-difusas. Revista Inf. Tecnol. 22(6), 111-1120 (2011).

Pérez M. A, y J. Vernon., Sistemas de lógica difusa (2005). Control Systems Principles. http://www.controlsystems-principles.co.uk/whitepapers/spanishwp/13fuzzylogicSP.pdf.Acceso: 1 Agosto (2013).

Salam M.A., S.M. Al-Alawi y A.A. Maqrashi., An Artificial neural network approach to model and predict the relationship between the grounding resistance and length of buried electrode in the soil. Science Direct. Journal of Electrostatics: 64(1), 338-342. (2006).

Sanchez, O.L., J. Velasco y C.A. Lozano., Diseño de una aplicación para la gestión de carga en transformadores de distribución. Revista Ingeniería e Investigación: 26 (3), 85-92 (2006).

Schau H. y A. Novitskiy., Economic transformer load estimation considering power quality. $13^{\text {th }}$ International Conference on Harmonics and Quality of Power (ICHQP), Wollongong, Australia, 1-6 (2008).

Stanley J., Introduction to Neural Networks, 3a edición. California Scientific Software, Sierra Madre, California, (1990).

Velilla, E., F. Villada, F. Echeverría., Modelos de pérdida de masa de acero por corrosión atmosférica en Colombia usando inteligencia computacional: Revista Facultad de Ingeniería Universidad de Antioquia: 49(1), 81-89 (2009).

Villada, F., A. Orille y J. Valencia., Aplicación de las Redes Neuronales en la Protección Diferencial de Generadores Sincrónicos. Revista Inf. Tecnol. 17(5), 83-90 (2006).

Villada, F., N. Muñoz y E. García., Aplicación de las redes neuronales al pronóstico de precios en el mercado de valores. Revista Inf. Tecnol. 23(4), 11-20 (2012).

Wang, P. ; Huang, J.Y. ; Ding, Y. ; Loh, P. ; Goel, L., Demand side load management of smart grids using intelligent trading/metering/ billing system. IEEE Power Tech conference, Trondhein, Norway, 1-6 (2011). 
